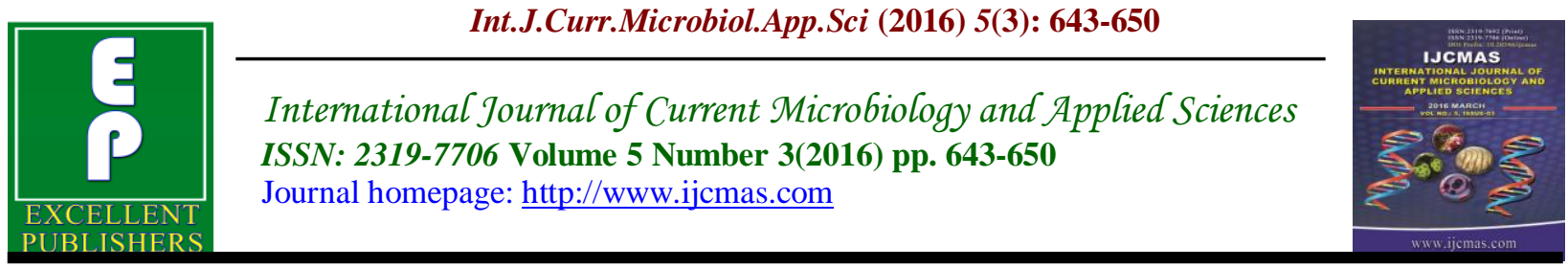

Original Research Article

http://dx.doi.org/10.20546/ijcmas.2016.503.075

\title{
Detection of biofilm formation among the clinical isolates of Enterococci: An evaluation of three different screening methods
}

\author{
L. Triveda* and S. Gomathi \\ Department of microbiology SRM Medical College Hospital and Research Centre \\ Kattankulathur, Tamilnadu, India \\ *Corresponding author
}

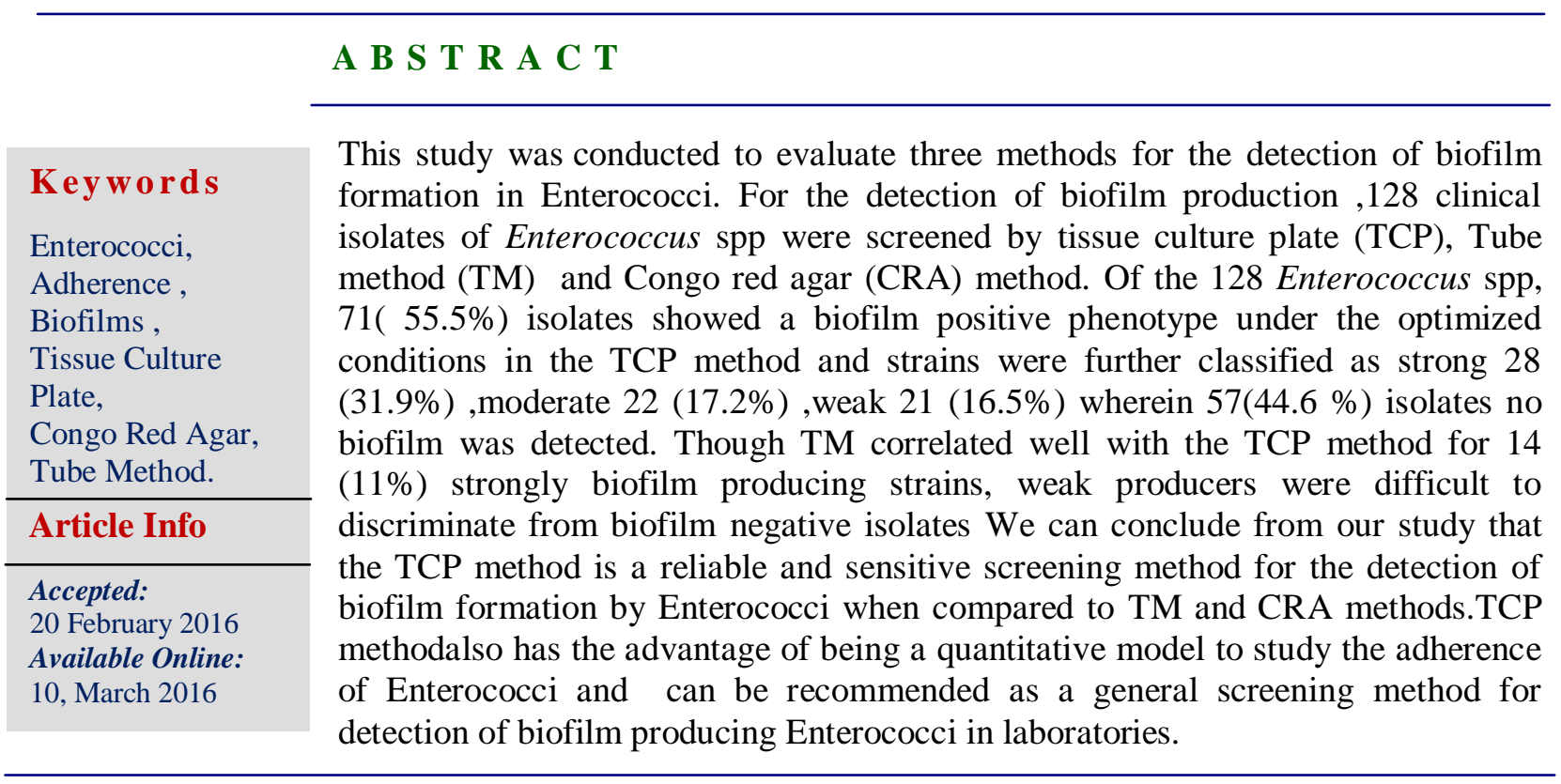

\section{Introduction}

Enterococci are Gram-positive member of the human gastrointestinal flora, and are also an important cause of opportunistic nosocomial infections [1]. These organisms are capable of infecting numerous body sites, causing bacteremia, intra-abdominal infections, endocarditis, and urinary tract infections [2]. Enterococcus faecalis and Enterococcus faecium are the most common enterococci species, and they are responsible for up to $95 \%$ of human enterococcal infections [3-5].
Biofilms are defined as microbially derived sessile communities characterized by the cells that are irreversibly attached to a substratum or to each other. They are embedded in a matrix of extracellular polymeric substances (EPS) they have produced, and exhibit an altered phenotype with respect to growth rate and gene transcription [6]. Within a biofilm, bacteria communicate with each other by production of chemotactic particles or pheromones, a phenomenon called quorum sensing 
[7]. Availability of key nutrients, chemotaxis towards surface, motility of bacteria, surface adhesins and presence of surfactants are some factors which influence biofilm formation. The ability of enterococci to form biofilms may confer an ecological advantage in certain situations. For example, clinical strains of $E$. faecalis isolated from infective endocarditis patients were significantly associated with the greater biofilm formation than nonendocarditis clinical isolates [8]; this may be attributable in part to specific virulence factors in enterococci [9]. Several enterococcal virulence factors have been identified, including adhesions and secreted virulence factors.

The most important adhesion factors are Asa (aggregation substance), Esp (extracellular surface protein), EfaA (E. faecalis antigen A), Ace (adhesin of collagen from $E$. faecalis) and Ebp (endocarditis and biofilmassociated pili) [10]. Several studies investigated the role of these virulence factors in biofilm formation by enterococci [11-14]. A number of tests are available to detect biofilm production. These include the Tissue culture plate (TCP), Tube method (TM), Congo Red Agar (CRA), Bioluminescent assay, piezoelectric sensors, and light and fluorescent microscopic examination. But the methods described latter are beyond the scope of many laboratories and hospitals.

The main objectives of this study was conducted to detect biofilm formation among clinical isolates of Enterococcus spp by three different methods - TCP(standard and modified), TM and CRA and also evaluated the reliability of these methods in order to determine the most suitable screening method for the detection of Enterococcal biofilms.

\section{Materials and Methods}

\section{Place and Duration of Study}

The study was conducted at the Department of Microbiology, SRM Medical college Hospital and Research Centre from April 2013 to March 2014.

\section{Selection of the Isolates}

A total of 128 non-repetitive clinical isolates of Enterococcus spp were included in this study. The samples from which these isolates were obtained include sterile body fluids like blood, peritoneal fluid, and isolates from urine, pus ,tissue and endotracheal tube samples. Samples were directly inoculated on blood agar, MacConkey agar, and CHROM agar, as per the nature of the specimen. Colony morphology and culture characteristics were observed macroscopically. Identification of genus Enterococcus was done based on Gram staining, cultural characteristics, and physiological and biochemical tests, namely, bile esculin hydrolysis, PYR hydrolysis, and growth in $6.5 \%$ sodium chloride and at $\mathrm{pH}$ $9.6[15]$

Reference strain of Enterococcus faecalis ATCC 29212 was included as control in our study.

\section{Detection of Biofilm Formation}

\section{Biofilm Production}

Biofilm production was studied employing three different methods. Three different methods, namely the tissue culture plate method, tube method and the congo red agar method were used. Though congo red agar is used for studying biofilms of Coagulase Negative Staphylococcus aureus (CONS), this medium was included in this study to 
see if it correlated with the findings obtained by other methods.

\section{Tissue Culture Plate Method (TCP)}

The TCP Assay described by Christensen et al [16] is most widely used and considered the gold-standard method for the detection of biofilm formation.In the present study we screened all the isolates for their ability to form biofilms as mentioned by Christensen et al [16] but with the modification of extended incubation period upto 24 hours .Many reports indicated the influence of media composition on biofilms, henceforth we evaluated biofilm production in three different media, Trypticase soy broth (TSB), Trypticase soy broth with $1 \%$ glucose $\left(\mathrm{TSB}_{\mathrm{glu}}\right)$ and Brain heart infusion broth with $2 \%$ sucrose (BHI suc) Colonies of Enterococci after overnight growth on blood agar were inoculated in repective media and incubated at $37^{\circ} \mathrm{C}$ in stationary condition .The broth culture was diluted 1:10 with freshly prepared medium. $20 \mu 1$ of broth culture was added to flat bottomed microtitre plate containing $230 \mu \mathrm{l}$ of TSB.A 96 well microtitre plate with flat bottom was used. First three wells served as media controls without the addition of cultures. 3 known in house positive and negative controls were inoculated in each plate. The test organism diluted in trypticase soy broth was inoculated in triplicate and incubated overnight at $37^{\circ} \mathrm{C}$ aerobically. After 24 hours of incubation,the plates were gently washed with PBS ( $\mathrm{pH}$ 7.2) thrice, to remove the free floating planktonic bacteria.300 $\mu$ l of methanol was added to each well and allowed to stand for 15 minutes. The excess of methanol was discarded, wells were stained using $0.1 \%$ Safranin stain. After 20 minutes of staining, excess stain was discarded and washed with phosphate buffer saline. The stained cells adherent to wells is resolubilised with $33 \%$ glacial acetic acid.
Test was carried out in triplicate and average of three optical density values were taken. The optical density of each well was measured at 490nm using automated Biorad Laboratory reader. The OD readings were considered as an index of bacteria adhering to surface and forming biofilms

\section{Tube Method (TM)}

A quantitative assessment of biofilm formation was described by Christensen et al [18]. Trypticase soy broth with $2 \%$ sucrose was inoculated with loopful of microorganisms from overnight culture and incubated for 24 hours at $37^{\circ} \mathrm{C}$. The tubes were then decanted and washed thrice with PBS pH 7.2 to remove any non - adherent cells. The tubes were air dried and stained with $0.1 \%$ crystal violet for 30 minutes. Excess stain was washed off with deionised water .The tubes were dried in inverted position and observed for biofilm production. Biofilm formation was considered positive when a visible film lines the sides and bottom of each tube. Tubes were then examined and the amount of biofilm was scored as 0-absent,1-weak,2moderate and 3-strong.Ring formation at the liquid interface was not indicative of biofilm formation. Experiment was performed in triplicate and repeated thrice.

\section{Congo Red Agar Method (CRA)}

Freeman et al [19] have described a simple qualitative method to detect biofilm production using Congo Red Agar (CRA) medium. The Congo red test is based on the ability of this dye to stain polysaccharides black. For preparing Congo Red Agar, sucrose and congo red stain $(50 \mathrm{~g} / \mathrm{L}$ and $0.8 \mathrm{~g} / \mathrm{L})$ were added to Brain Heart Infusion agar. Enterococcal strains were inoculated on the Congo Red Agar plates and incubated at $37^{\circ} \mathrm{C}$ for 24 hours.A positive result was 
indicated by black crystalline morphology. Isolates producing black colonies were considered as Strong biofilm producers. Dark colonies without dry crystalline colony morphology indicted moderate biofilm production. Weak biofilm producers produced dark pink colonies. Non-slime producers mostly turned out as dry red colonies. The experiment was performed in triplicate and repeated thrice.

\section{Statistical Evaluation of TCP, TM and CRA Method for Detection of Biofilm Formation Among Clinical Isolates of Enterococci Spp}

The comparative statistical analysis for all methods was done by using Greenhalgh $2 \times 2$ table [20]. Data obtained from standard TCP method, considered gold standard was compared with data from TM and CRA methods. Parameters like sensitivity: $a /(a+c), \quad$ specificity : $d /(b+d)$, negative predictive value $(\mathrm{PV}-): \mathrm{d} /(\mathrm{c}+\mathrm{d})$, positive predictive value $(\mathrm{PV}+): \mathrm{a} /(\mathrm{a}+\mathrm{b})$ were determined, wherein $\mathrm{a}, \mathrm{b}, \mathrm{c}$, and $\mathrm{d}$ refer to the number of determinants in which true positives (a) were biofilm producers by standard TCP method( gold standard) as well as positive by TM and CRA (b) were biofilm producers by TM and CRA method but negative by TCP method, false negatives (c) were non biofilm producers by TM and CRA but biofilm producers by standard TCP, true negatives and (d) were non biofilm producers by all the methods.

\section{Results and Discussion}

In the standard TCP assay, from the total number of 128 isolates tested for biofilm formation,only $4(3.2 \%)$ isolates displayed strong biofilm phenotype in TSB medium, whereas with the addition of $1 \%$ glucose in TSB ( TSB $_{\text {glu }}$ ),number of biofilm forming isolates increased to $41(32.03 \%)$ and 71
(55.5\%) after incubation for 18 hour and 24 hours respectively. Similarly ,by using BHI $_{\text {suc }}$ medium 68 (53.2\%) biofilm forming isolates were detected.

In the modified TCP method, from the total number of 128 isolates tested for biofilm production, strong biofilm producers were $28(31.9 \%), 22(17.2 \%)$ were moderate and $21(16.5 \%)$ were weak and $57(44.6 \%)$ isolates were considered as non biofilm producers.

The TM showed good correlation with the TCP assay for strong biofilm forming isolates and total $14(11 \%)$ isolates were picked up as strong and $14(11 \%)$ were moderate biofilm producers. $10(7.8 \%)$ were identified as weak biofilm producers in contrast to 90 ( $70.4 \%)$ of non-biofilm producers. However, it was difficult to discriminate between moderate and weakly biofilm producing isolates.

By CRA method we obtained very different results, most of the strains displayed red colonies (pink to orange ) , 11(8.6\%) isolates showed black colonies, but no typical crystalline morphology was observed. Only $5(4 \%)$ isolates displayed pink-red colonies without dry crystalline morphology after $24-48$ hours. 10 (7.8\%) isolates showed pink colony morphology 102 (79.7\%) isolates displayed dry red colony morphology after 24-48 hours. However, among these isolates identified as non -slime producers ,around15( 14.7\%) isolates displayed slight black discolouration without crystalline morphology,no correlation between colony morphology on CRA and TCP results was observed .

Using well- characterized 128 isolates data obtained from standard TCP method was used to evaluate TM and CRA methods. The TCP method was most sensitive and specific 
in terms of discriminating between biofilm producers and non-biofilm producers. In TM, strong biofilm producers could be very well detected, whereas it was very difficult to distinguish between moderate and weak biofilm forming isolates, thereby affecting its performance in terms of sensitivity
$(46.9 \%)$,specificity $(82.2 \%)$.

CRA method showed very little correlation with corresponding methods and the parameters of sensitivity (25\%), specificity $(46.7 \%)$ were very low.

Table.1 Interpretation [17]

\begin{tabular}{|l|l|l|}
\hline S.No & OD Value & Biofilm \\
\hline 1 & $<0.314$ & Non adherent \\
\hline 2 & $0.314-0.628$ & Weak \\
\hline 3 & $0.628-1.256$ & Moderate \\
\hline 4 & $>1.256$ & Strong \\
\hline
\end{tabular}

Table.2 Screening of 128 Enterococcal Isolates for Biofilm Formation by TCP Method and at 18 and 24 Hours of Incubation

\begin{tabular}{|c|c|c|c|c|c|c|}
\hline \multirow[t]{4}{*}{ Biofilm formation (OD $\left.{ }_{490 \mathrm{~nm}}\right)$} & \multicolumn{6}{|c|}{ No of isolates } \\
\hline & \multicolumn{2}{|c|}{ TSB } & \multicolumn{2}{|c|}{$\mathbf{T S B}_{\text {glu }}$} & \multicolumn{2}{|c|}{$\mathbf{B H I}_{\text {suc }}$} \\
\hline & \multicolumn{6}{|c|}{ Incubation period (hour) } \\
\hline & 18 & 24 & 18 & 24 & 18 & 24 \\
\hline Strong (> 1.256) & 1 & 2 & 16 & 28 & 18 & 14 \\
\hline Moderate $(0.628-1.256)$ & 3 & 6 & 15 & 22 & 25 & 25 \\
\hline Weak $(0.314-0.628)$ & - & - & 10 & 21 & 60 & 29 \\
\hline Non Adherent $<\mathbf{0 . 3 1 4}$ & 124 & 120 & 87 & 57 & 60 & 60 \\
\hline
\end{tabular}

Table.3 Screening of 128 Enterococcal Isolates for Biofilm Formation by TCP,TM and CRA Method

\begin{tabular}{|c|c|c|c|c|c|}
\hline \multirow[t]{2}{*}{ No of Isolates } & \multirow{2}{*}{$\begin{array}{l}\text { Biofilm } \\
\text { formation }\end{array}$} & \multicolumn{4}{|c|}{ Screening Methods } \\
\hline & & & TCP & $\mathbf{T M}$ & CRA $^{b}$ \\
\hline \multirow[t]{4}{*}{ Clinical Isolates $\mathbf{n = 1 2 8}$} & Strong & & 28 & 14 & 11 \\
\hline & Moderate & & 22 & 14 & 5 \\
\hline & Weak & & 21 & 10 & 10 \\
\hline & Non Adherent & & 57 & 90 & 102 \\
\hline \multirow[t]{2}{*}{ Standard Isolates } & \multicolumn{5}{|c|}{ Biofilm formation } \\
\hline & \multicolumn{2}{|c|}{ TCP } & \multicolumn{2}{|c|}{ TM } & CRA \\
\hline E.faecalis ATCC 29212 & \multicolumn{2}{|c|}{ Strong $\left(\mathrm{OD}_{490}>1.256\right)$} & \multicolumn{2}{|c|}{ Strong (Score 3) } & $\begin{array}{l}\text { Moderate (Dark } \\
\text { Colonies with dry } \\
\text { crystalline } \\
\text { morphology) }\end{array}$ \\
\hline
\end{tabular}


Table.4 Statistical Evaluation of Modified TCP , TM, CRA Methods for Detection of Biofilm Formation in Enterococcal $(n=152)$

\begin{tabular}{|l|c|c|c|c|}
\hline \multicolumn{5}{|c|}{ Test Characteristics(\%) } \\
\hline Screening Method & Sensitivity & Specificity & $\begin{array}{c}\text { Positive predictive } \\
\text { value }\end{array}$ & $\begin{array}{c}\text { Negative predictive } \\
\text { value }\end{array}$ \\
\hline Tissue culture plate & 87.5 & 85.07 & 73.68 & 98.25 \\
\hline Tube Method & 45.9 & 82.14 & 42.31 & 67.65 \\
\hline Congo red agar & 25 & 46.67 & 9.86 & 29.9 \\
\hline
\end{tabular}

Figure.1 Screening of Biofilm Producers by TCP Method

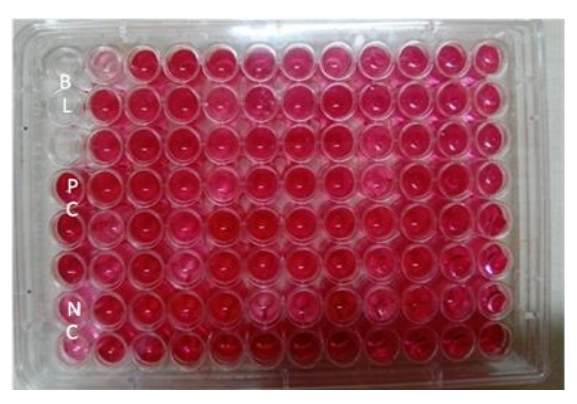

Figure.2 Screening of Biofilm Producers by Tube Method

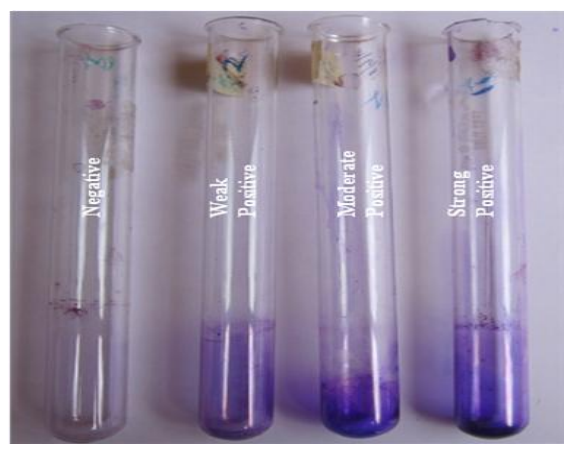

Figure.3 Screening of Biofilm Producers by Congo Red Agar Medium

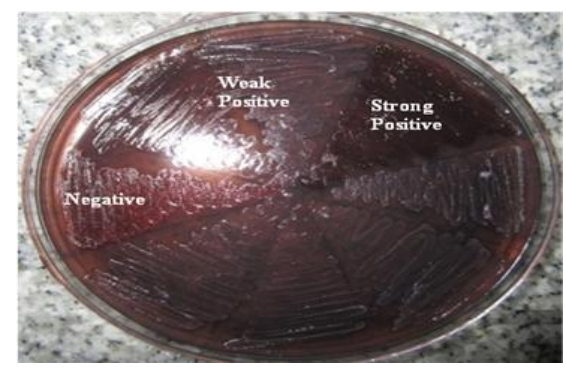


Adherence to body surfaces is considered a major factor responsible for the pathogenicity of clinical isolates of Enterococcus species. Biofilm formation plays a major role in nosocomial infections like catheter-associated UTIs and even blood stream infections due to pacemakers. It also plays a vital role in endodontic infections. Investigations to understand the pathogenesis of these infections have focused upon the process of adherence of these microorganisms on these devices and have used various methods to quantify number of organisms adhering to surface [16-23]. We tested 128 clinical isolates of Enterococci by three in vitro screening procedures for their ability to form biofilm.

In the TCP assay with TSB medium only 4 (3.2\%) of 128 tested Enterococci isolates showed biofilm positive phenotype. This was in agreement with the observations of other investigators in which few or no biofilm producing isolates could be detected using this medium [ 20]. On the otherhand, supplementation of TSB and BHI media with different sugars such as glucose and sucrose exhibited biofilm formation in 68 $(53.2 \%)$ and $41(32 \%)$ isolates respectively as investigated in a study by Ira et al [24 ].

In the modified TCP method, extended incubation for 24 hour could lead to a better discrimination between moderate and nonbiofilm producing Enterococci and biofilm formation was observed in $71(55.5 \%)$ isolates. These observations suggested a strong dependence between biofilm formation in Enterococci and that the use of various sugar supplementation

The tube method correlates well with the TCP test for strongly biofilm producing isolates but it was difficult to discriminate between weak biofilm producing and non adherent isolates due to observer bias.
Consequently ,high variability was observed and classification in biofilm positive and negative was difficult by tube method. In agreement with the previous reports ,tube test cannot be recommended as a general screening test to identify biofilm producing isolates [24-25]

Congo red agar in our study was prepared by combining Brain heart infusion agar with $5 \%$ sucrose and $0.8 \%$ of congo red dye. This is in compliance with the formulation suggested by Mathur et al in their study [25]. In CRA method, out of positive isolates, $11(8.6 \%)$ displayed black colonies with no dry crystalline morphology . Only 5 (4\%) isolates displayed pink-red colonies without dry crystalline morphology . 10 (7.8\%) isolates showed pink colony morphology , $102 \quad(79.7 \%)$ isolates displayed dry red colony morphology after 24-48 hours. However, among these isolates identified as non -slime producers , around $15(14.7 \%)$ isolates displayed slight black discolouration without crystalline morphology, hence no correlation between colony morphology on CRA and TCP results was observed.Congo red agar poorly detected Enterococcal biofilms which was entirely in agreement with the observation made by Parharaj et al [24 ]. CRA though specific for the detection of biofilms produced by Staphylococcus species, has been included in our study to see, if this medium correlated with the findings obtained by other methods.Based on our results we are unable to recommend the CRA method for the detection of biofilm formation by Enterococcus isolates.

Our data indicates that the TCP method is accurate and reproducible method for screening and this technique can serve as a reliable quantitative tool for determining biofilm formation by clinical isolates of Enterococci. 


\section{References}

1. Sandoe, J.A., Witherden, I.R., Cove, J.H., Heritage, J., Wilcox, M.H., 2003. Correlation between enterococcal biofilm formation in vitro and medical-devicerelated infection potential in vivo. J. Med. Microbiol. 52, 547-550.

2. Shankar, V., Baghdayan, A.S., Huycke, M.M., Lindahl, G., Gilmore, M.S., 1999. Infection-derived Enterococcus faecalis strains are enriched in esp, a gene encoding a novel surface protein. Infect. Immun. 67, 193-200.

3. Shankar, N., Lockatell, C.V., Baghdayan, A.S., Drachenberg, C., Gilmore, M.S., Johnson, D.E., 2001. Role of Enterococcus faecalis surface protein Esp in the pathogenesis of ascending urinary tract infection. Infect. Immun. 69, 4366-4372

4. Dupre, I., Zanetti, S., Schito, A.M., Fadda, G., Sechi, L.A., 2003. Incidence of virulence determinants in clinical Enterococcus faecium and Enterococcus faecalis isolates collected in Sardinia (Italy). J. Med. Microbiol. 52, 491-498

5. Winn W, Allen S, Janda W, et al. Charts. In : Koneman's Color Atlas and Text Book of Diagnostic Microbiology, $6^{\text {th }}$ Edition, Philadelphia USA:Lippincott Williams and Wilkins; 2006.1442-1535.

6. Christensen GD, Simpson WA, Younger JA, Baddour LM, Barrett FF, Melton DM, et al. Adherence of cogulase negative Staphylococi to plastic tissue cultures:a quantitative model for the adherence of staphylococci to medical devices. J ClinMicrobiol 1985;22:996-1006

7. Stepanovic S, Cirkovic I, Ranin L and Svabic -VlahovicM.Biofilm formation by Salmonella spp and Listeria monocytogenes on plastic surface.Letter in Applied Microbiology 2004;38(5):428-432 .

8. Christensen GD, Simpson WA, Bisno AL, Beachey EH. Adherence of slimeproducing strains of Staphylococcus epidermidis to smooth surfaces. Infect Immun 1982;37:318-26

9. Freeman DJ, Falkiner FR, Keane CT. New method for detecting slime production by coagulase negative staphylococci. J ClinPathol 1989;42:872-4.

10. Johannes KM, Knobloch M, Matthias A, Rohde H, Mack D. Evaluation of different methods of biofilm formation in Staphylococcus aureus. Med MicrobiolImmunol 2002;191:101- 6 .

11. Ludwicka A, Switalski LM, Lundin A, Pulverer G, Wadstrom T. Bioluminescent assays for measurement of bacterial attachment to polyethylene. J Microbiol Methods 1985;4:169- 77.

12. Donlan RM, Murga R, Bell M, Toscano CM, Carr JH, Novicki TJ, et al. Protocol for detection of biofilms on needleless connectors attached to central venous catheters. J ClinMicrobiol 2001;39:750-3.

13. Zufferey J, Rime B, Francioli P, Bille J. Simple method for rapid diagnosis of Catheter-associated infection by direct Acridine orange staining of catheter tips. J ClinMicrobiol 1988;26:175-7

14. Ira P, Sujatha S, Chandra PS. Virulence factors in clinical and commensal isolates of Enterococcus species. Ind $\mathbf{J}$ PatholMicrobiol 2013;56:24-30.

15. Mathur T, Singhal S, Khan S, Upadhyay DJ, Fatma T, Rattan A. Detection of biofilm formation among the clinical isolates of staphylococci:an evaluation of three different screening methods. Ind $\mathbf{J}$ Med Microbiol 2006;24(1):25-9

\section{How to cite this article:}

Triveda, L., and S. Gomathi. 2016. Detection of biofilm formation among the clinical isolates of Enterococci: An evaluation of three different screening methods. Int.J.Curr.Microbiol.App.Sci. 5(3): 643-650. doi: http://dx.doi.org/10.20546/ijcmas.2016.503.075 